\title{
A Side Reaction of Significance in Preparation of Peptide- or Peptidomimetic-Based Hydroxamate Enzyme Inhibitors
}

\author{
Dusan Hesek, Bruce C. Noll, and Shahriar Mobashery* \\ Department of Chemistry and Biochemistry and the Walther Cancer Research Center, \\ University of Notre Dame, Notre Dame, IN 45665 \\ mobashery@nd.edu
}

Supporting Information

Table of Contents.

Table 1. Crystal data and structure refinement for $\mathrm{C}_{19} \mathrm{H}_{24} \mathrm{~N}_{2} \mathrm{O}_{3}, \mathbf{8} \ldots \ldots \ldots \ldots \ldots$ S2

Table 2. Atomic coordinates and equivalent isotropic displacement parameters for 8 S3

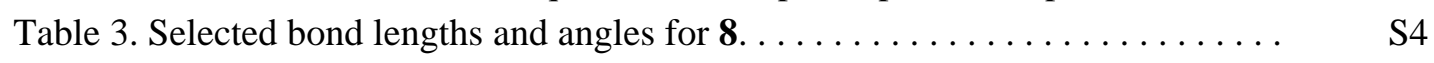

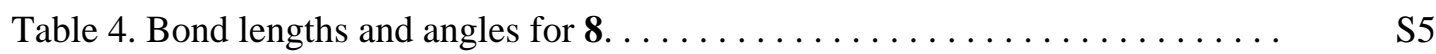

Table 5. Anisotropic displacement parameters for $8 \ldots \ldots \ldots \ldots \ldots \ldots \ldots$ S7

Table 6. Hydrogen coordinates and isotropic displacement parameters for $8 \ldots \ldots$ S8

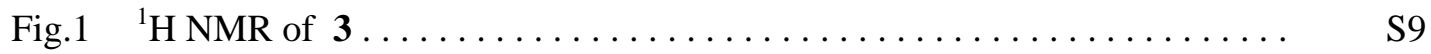

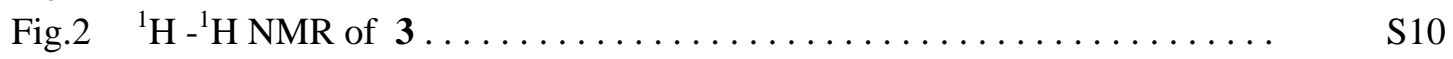

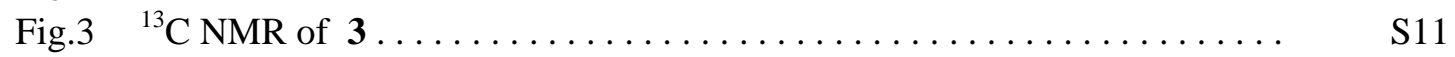

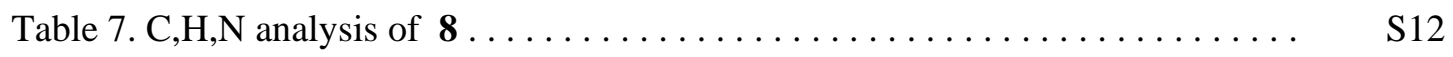

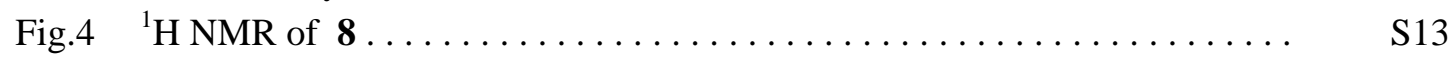

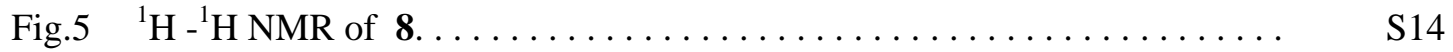

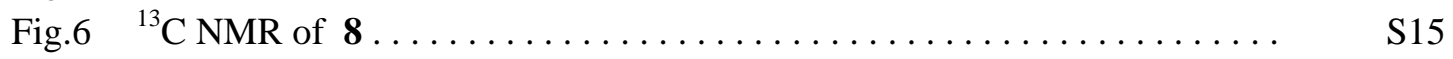

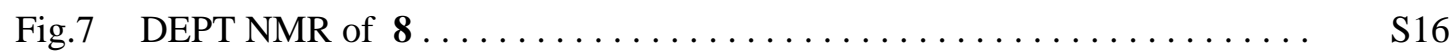

General Experimental Methods $\ldots \ldots \ldots \ldots \ldots \ldots \ldots \ldots \ldots \ldots \ldots \ldots \ldots \ldots \ldots \ldots \ldots \ldots$

CIF for $8 . \ldots \ldots \ldots \ldots \ldots \ldots \ldots \ldots \ldots \ldots \ldots \ldots \ldots \ldots \ldots \ldots \ldots \ldots \ldots \ldots \ldots \ldots \ldots \ldots$ 
Table 1. Crystal data and structure refinement for $\mathrm{C}_{19} \mathrm{H}_{24} \mathrm{~N}_{2} \mathrm{O}_{3}, \mathbf{8}$.

Identification code

Empirical formula

Formula weight

Temperature

Wavelength

Crystal system

Space group

Unit cell dimensions

Volume

Z

Density (calculated)

Absorption coefficient

$\mathrm{F}(000)$

Crystal size

Crystal color and habit

Diffractometer

$\theta$ range for data collection

Index ranges

Reflections collected

Independent reflections

Observed reflections $(\mathrm{I}>2 \sigma(\mathrm{I}))$

Completeness to $\theta=31.57^{\circ}$

Absorption correction

Max. and min. transmission

Solution method

Refinement method

Data / restraints / parameters

Goodness-of-fit on $\mathrm{F}^{2}$

Final $R$ indices $[I>2 \sigma(I)]$

$\mathrm{R}$ indices (all data)

Absolute structure parameter

Largest diff. peak and hole bcn172_0m

C19 H24 N2 O3

328.40

100(2) K

$0.71073 \AA$

monoclinic

P 21

$\mathrm{a}=7.3650(15) \AA$

$\alpha=90^{\circ}$.

$\mathrm{b}=12.969(3) \AA$

$\beta=96.68(3)^{\circ}$

$\mathrm{c}=9.831(2) \AA$

$\gamma=90^{\circ}$.

932.7(3) $\AA^{3}$

2

$1.169 \mathrm{Mg} / \mathrm{m}^{3}$

$0.079 \mathrm{~mm}^{-1}$

352

$0.57 \times 0.45 \times 0.28 \mathrm{~mm}^{3}$

clear colorless plate

Bruker SMART APEX CCD diffractometer

2.09 to $31.57^{\circ}$.

$-10<=\mathrm{h}<=10,-19<=\mathrm{k}<=19,-14<=\mathrm{k}<=14$

46162

$3208[\mathrm{R}(\mathrm{int})=0.0273]$

3178

$99.3 \%$

None

0.98 and 0.97

SHELXS-97 (Sheldrick, 1990)

SHELXL-97 (Sheldrick, 1997)

$3208 / 1 / 225$

1.071

$\mathrm{R} 1=0.0301, \mathrm{wR} 2=0.0845$

$\mathrm{R} 1=0.0303, \mathrm{wR} 2=0.0849$

$0(10)$

0.350 and $-0.193 \mathrm{e} \cdot \AA^{-3}$ 
Table 2. Atomic coordinates and equivalent isotropic displacement parameters $\left(\AA^{2}\right)$ for $\mathbf{8}$. $U(\mathrm{eq})$ is defined as one third of the trace of the orthogonalized $U_{i j}$ tensor.

\begin{tabular}{lllll} 
& $\mathrm{x}$ & $\mathrm{y}$ & \multicolumn{1}{c}{$\mathrm{z}$} & $\mathrm{U}(\mathrm{eq})$ \\
$\mathrm{N}(1)$ & $0.99703(12)$ & $0.11178(7)$ & $0.34913(8)$ & $0.01904(16)$ \\
$\mathrm{N}(2)$ & $0.74696(11)$ & $0.17279(7)$ & $0.50852(8)$ & $0.01708(15)$ \\
$\mathrm{O}(1)$ & $0.80480(12)$ & $0.13300(9)$ & $0.15411(8)$ & $0.0325(2)$ \\
$\mathrm{O}(2)$ & $0.44972(9)$ & $0.11880(6)$ & $0.53883(7)$ & $0.01784(14)$ \\
$\mathrm{O}(3)$ & $0.21106(11)$ & $0.05959(7)$ & $0.63974(9)$ & $0.02547(17)$ \\
$\mathrm{C}(1)$ & $0.69194(13)$ & $0.18315(8)$ & $0.36183(9)$ & $0.01739(17)$ \\
$\mathrm{C}(2)$ & $0.65899(14)$ & $0.29849(9)$ & $0.32623(11)$ & $0.02078(18)$ \\
$\mathrm{C}(3)$ & $0.48402(14)$ & $0.33765(8)$ & $0.37332(11)$ & $0.02011(18)$ \\
$\mathrm{C}(4)$ & $0.47743(15)$ & $0.37025(9)$ & $0.50811(12)$ & $0.02286(19)$ \\
$\mathrm{C}(5)$ & $0.31231(17)$ & $0.40105(9)$ & $0.55186(14)$ & $0.0279(2)$ \\
$\mathrm{C}(6)$ & $0.15283(17)$ & $0.40117(10)$ & $0.46079(16)$ & $0.0310(3)$ \\
$\mathrm{C}(7)$ & $0.15817(16)$ & $0.37043(10)$ & $0.32601(15)$ & $0.0302(2)$ \\
$\mathrm{C}(8)$ & $0.32266(15)$ & $0.33847(9)$ & $0.28261(12)$ & $0.0246(2)$ \\
$\mathrm{C}(9)$ & $0.83857(13)$ & $0.14023(9)$ & $0.27969(9)$ & $0.01887(18)$ \\
$\mathrm{C}(10)$ & $1.14170(15)$ & $0.06777(10)$ & $0.27930(11)$ & $0.0248(2)$ \\
$\mathrm{C}(11)$ & $0.63071(13)$ & $0.14336(7)$ & $0.58509(9)$ & $0.01624(16)$ \\
$\mathrm{C}(12)$ & $0.66219(13)$ & $0.12770(8)$ & $0.73448(9)$ & $0.01690(16)$ \\
$\mathrm{C}(13)$ & $0.50489(14)$ & $0.09172(8)$ & $0.77464(10)$ & $0.01930(18)$ \\
$\mathrm{C}(14)$ & $0.36867(14)$ & $0.08601(8)$ & $0.65163(10)$ & $0.01920(18)$ \\
$\mathrm{C}(15)$ & $0.84173(13)$ & $0.15137(8)$ & $0.81478(10)$ & $0.01891(17)$ \\
$\mathrm{C}(16)$ & $0.87788(15)$ & $0.26863(9)$ & $0.82851(10)$ & $0.02208(19)$ \\
$\mathrm{C}(17)$ & $1.0682(2)$ & $0.28630(13)$ & $0.90481(15)$ & $0.0382(3)$ \\
$\mathrm{C}(18)$ & $0.7312(2)$ & $0.32166(10)$ & $0.90187(13)$ & $0.0306(2)$ \\
$\mathrm{C}(19)$ & $0.45912(17)$ & $0.05869(11)$ & $0.91175(11)$ & $0.0279(2)$
\end{tabular}


Table 3. Selected bond lengths $[\AA]$ and angles $\left[^{\circ}\right]$ for 8.

$\begin{array}{lllr}\mathrm{N}(2)-\mathrm{C}(11) & 1.2630(13) & \mathrm{N}(2)-\mathrm{C}(1) & 1.4582(12) \\ \mathrm{O}(2)-\mathrm{C}(14) & 1.3860(12) & \mathrm{O}(2)-\mathrm{C}(11) & 1.3943(12) \\ \mathrm{O}(3)-\mathrm{C}(14) & 1.2028(13) & \mathrm{C}(1)-\mathrm{C}(9) & 1.5267(14) \\ \mathrm{C}(1)-\mathrm{C}(2) & 1.5490(15) & \mathrm{C}(11)-\mathrm{C}(12) & 1.4740(13) \\ \mathrm{C}(12)-\mathrm{C}(13) & 1.3499(14) & \mathrm{C}(13)-\mathrm{C}(14) & 1.4807(15) \\ \mathrm{C}(13)-\mathrm{C}(19) & 1.4899(14) & & \\ & & & \\ \mathrm{C}(14)-\mathrm{O}(2)-\mathrm{C}(11) & 107.07(8) & \mathrm{N}(2)-\mathrm{C}(1)-\mathrm{C}(9) & 110.93(8) \\ \mathrm{N}(2)-\mathrm{C}(1)-\mathrm{C}(2) & 109.31(8) & \mathrm{C}(9)-\mathrm{C}(1)-\mathrm{C}(2) & 109.54(8) \\ \mathrm{N}(2)-\mathrm{C}(11)-\mathrm{O}(2) & 124.28(9) & \mathrm{N}(2)-\mathrm{C}(11)-\mathrm{C}(12) & 126.62(9) \\ \mathrm{O}(2)-\mathrm{C}(11)-\mathrm{C}(12) & 109.10(8) & \mathrm{C}(12)-\mathrm{C}(13)-\mathrm{C}(14) & 107.55(9) \\ \mathrm{C}(12)-\mathrm{C}(13)-\mathrm{C}(19) & 131.01(10) & \mathrm{C}(14)-\mathrm{C}(13)-\mathrm{C}(19) & 121.43(9)\end{array}$


Table 4. Bond lengths $[\AA]$ and angles $\left[{ }^{\circ}\right]$ for 8 .

\begin{tabular}{|c|c|c|c|}
\hline $\mathrm{N}(1)-\mathrm{C}(9)$ & $1.3337(13)$ & $\mathrm{N}(1)-\mathrm{C}(10)$ & $1.4500(13)$ \\
\hline $\mathrm{N}(1)-\mathrm{H}(1 \mathrm{~N})$ & $0.854(18)$ & $\mathrm{N}(2)-\mathrm{C}(11)$ & $1.2630(13)$ \\
\hline $\mathrm{N}(2)-\mathrm{C}(1)$ & $1.4582(12)$ & $\mathrm{O}(1)-\mathrm{C}(9)$ & $1.2343(12)$ \\
\hline $\mathrm{O}(2)-\mathrm{C}(14)$ & $1.3860(12)$ & $\mathrm{O}(2)-\mathrm{C}(11)$ & $1.3943(12)$ \\
\hline $\mathrm{O}(3)-\mathrm{C}(14)$ & $1.2028(13)$ & $\mathrm{C}(1)-\mathrm{C}(9)$ & $1.5267(14)$ \\
\hline $\mathrm{C}(1)-\mathrm{C}(2)$ & $1.5490(15)$ & $\mathrm{C}(1)-\mathrm{H}(1)$ & 1.0000 \\
\hline $\mathrm{C}(2)-\mathrm{C}(3)$ & $1.5072(14)$ & $\mathrm{C}(2)-\mathrm{H}(2 \mathrm{~A})$ & 0.9900 \\
\hline $\mathrm{C}(2)-\mathrm{H}(2 \mathrm{~B})$ & 0.9900 & $C(3)-C(4)$ & $1.3971(16)$ \\
\hline$C(3)-C(8)$ & $1.4004(15)$ & $C(4)-C(5)$ & $1.3947(16)$ \\
\hline $\mathrm{C}(4)-\mathrm{H}(4)$ & 0.9500 & $C(5)-C(6)$ & $1.3916(19)$ \\
\hline $\mathrm{C}(5)-\mathrm{H}(5)$ & 0.9500 & $C(6)-C(7)$ & $1.389(2)$ \\
\hline $\mathrm{C}(6)-\mathrm{H}(6)$ & 0.9500 & $C(7)-C(8)$ & $1.3934(16)$ \\
\hline $\mathrm{C}(7)-\mathrm{H}(7)$ & 0.9500 & $\mathrm{C}(8)-\mathrm{H}(8)$ & 0.9500 \\
\hline $\mathrm{C}(10)-\mathrm{H}(10 \mathrm{~A})$ & 0.9800 & $\mathrm{C}(10)-\mathrm{H}(10 \mathrm{~B})$ & 0.9800 \\
\hline $\mathrm{C}(10)-\mathrm{H}(10 \mathrm{C})$ & 0.9800 & $\mathrm{C}(11)-\mathrm{C}(12)$ & $1.4740(13)$ \\
\hline $\mathrm{C}(12)-\mathrm{C}(13)$ & $1.3499(14)$ & $C(12)-C(15)$ & $1.4911(14)$ \\
\hline$C(13)-C(14)$ & $1.4807(15)$ & $C(13)-C(19)$ & $1.4899(14)$ \\
\hline$C(15)-C(16)$ & $1.5470(16)$ & $\mathrm{C}(15)-\mathrm{H}(15 \mathrm{~A})$ & 0.9900 \\
\hline $\mathrm{C}(15)-\mathrm{H}(15 \mathrm{~B})$ & 0.9900 & $C(16)-C(17)$ & $1.5284(17)$ \\
\hline$C(16)-C(18)$ & $1.5302(17)$ & $\mathrm{C}(16)-\mathrm{H}(16)$ & 1.0000 \\
\hline $\mathrm{C}(17)-\mathrm{H}(17 \mathrm{~A})$ & 0.9800 & $\mathrm{C}(17)-\mathrm{H}(17 \mathrm{~B})$ & 0.9800 \\
\hline $\mathrm{C}(17)-\mathrm{H}(17 \mathrm{C})$ & 0.9800 & $\mathrm{C}(18)-\mathrm{H}(18 \mathrm{~A})$ & 0.9800 \\
\hline $\mathrm{C}(18)-\mathrm{H}(18 \mathrm{~B})$ & 0.9800 & $\mathrm{C}(18)-\mathrm{H}(18 \mathrm{C})$ & 0.9800 \\
\hline $\mathrm{C}(19)-\mathrm{H}(19 \mathrm{~A})$ & 0.9800 & $\mathrm{C}(19)-\mathrm{H}(19 \mathrm{~B})$ & 0.9800 \\
\hline $\mathrm{C}(19)-\mathrm{H}(19 \mathrm{C})$ & 0.9800 & & \\
\hline $\mathrm{C}(9)-\mathrm{N}(1)-\mathrm{C}(10)$ & $120.93(8)$ & $\mathrm{C}(9)-\mathrm{N}(1)-\mathrm{H}(1 \mathrm{~N})$ & $120.4(13)$ \\
\hline $\mathrm{C}(10)-\mathrm{N}(1)-\mathrm{H}(1 \mathrm{~N})$ & $118.4(13)$ & $\mathrm{C}(11)-\mathrm{N}(2)-\mathrm{C}(1)$ & $119.19(9)$ \\
\hline $\mathrm{C}(14)-\mathrm{O}(2)-\mathrm{C}(11)$ & $107.07(8)$ & $\mathrm{N}(2)-\mathrm{C}(1)-\mathrm{C}(9)$ & $110.93(8)$ \\
\hline $\mathrm{N}(2)-\mathrm{C}(1)-\mathrm{C}(2)$ & $109.31(8)$ & $\mathrm{C}(9)-\mathrm{C}(1)-\mathrm{C}(2)$ & $109.54(8)$ \\
\hline $\mathrm{N}(2)-\mathrm{C}(1)-\mathrm{H}(1)$ & 109.0 & $\mathrm{C}(9)-\mathrm{C}(1)-\mathrm{H}(1)$ & 109.0 \\
\hline $\mathrm{C}(2)-\mathrm{C}(1)-\mathrm{H}(1)$ & 109.0 & $\mathrm{C}(3)-\mathrm{C}(2)-\mathrm{C}(1)$ & $111.91(8)$ \\
\hline $\mathrm{C}(3)-\mathrm{C}(2)-\mathrm{H}(2 \mathrm{~A})$ & 109.2 & $\mathrm{C}(1)-\mathrm{C}(2)-\mathrm{H}(2 \mathrm{~A})$ & 109.2 \\
\hline $\mathrm{C}(3)-\mathrm{C}(2)-\mathrm{H}(2 \mathrm{~B})$ & 109.2 & $\mathrm{C}(1)-\mathrm{C}(2)-\mathrm{H}(2 \mathrm{~B})$ & 109.2 \\
\hline $\mathrm{H}(2 \mathrm{~A})-\mathrm{C}(2)-\mathrm{H}(2 \mathrm{~B})$ & 107.9 & $\mathrm{C}(4)-\mathrm{C}(3)-\mathrm{C}(8)$ & $118.76(10)$ \\
\hline$C(4)-C(3)-C(2)$ & $121.23(10)$ & $\mathrm{C}(8)-\mathrm{C}(3)-\mathrm{C}(2)$ & $119.97(10)$ \\
\hline$C(5)-C(4)-C(3)$ & $120.45(11)$ & $\mathrm{C}(5)-\mathrm{C}(4)-\mathrm{H}(4)$ & 119.8 \\
\hline $\mathrm{C}(3)-\mathrm{C}(4)-\mathrm{H}(4)$ & 119.8 & $\mathrm{C}(6)-\mathrm{C}(5)-\mathrm{C}(4)$ & $120.19(12)$ \\
\hline $\mathrm{C}(6)-\mathrm{C}(5)-\mathrm{H}(5)$ & 119.9 & $\mathrm{C}(4)-\mathrm{C}(5)-\mathrm{H}(5)$ & 119.9 \\
\hline$C(7)-C(6)-C(5)$ & $119.88(11)$ & $\mathrm{C}(7)-\mathrm{C}(6)-\mathrm{H}(6)$ & 120.1 \\
\hline $\mathrm{C}(5)-\mathrm{C}(6)-\mathrm{H}(6)$ & 120.1 & $C(6)-C(7)-C(8)$ & $119.97(12)$ \\
\hline $\mathrm{C}(6)-\mathrm{C}(7)-\mathrm{H}(7)$ & 120.0 & $\mathrm{C}(8)-\mathrm{C}(7)-\mathrm{H}(7)$ & 120.0 \\
\hline $\mathrm{C}(7)-\mathrm{C}(8)-\mathrm{C}(3)$ & $120.73(11)$ & $\mathrm{C}(7)-\mathrm{C}(8)-\mathrm{H}(8)$ & 119.6 \\
\hline $\mathrm{C}(3)-\mathrm{C}(8)-\mathrm{H}(8)$ & 119.6 & $\mathrm{O}(1)-\mathrm{C}(9)-\mathrm{N}(1)$ & 123.61(10) \\
\hline $\mathrm{O}(1)-\mathrm{C}(9)-\mathrm{C}(1)$ & $118.95(9)$ & $\mathrm{N}(1)-\mathrm{C}(9)-\mathrm{C}(1)$ & $117.44(8)$ \\
\hline $\mathrm{N}(1)-\mathrm{C}(10)-\mathrm{H}(10 \mathrm{~A})$ & 109.5 & $\mathrm{~N}(1)-\mathrm{C}(10)-\mathrm{H}(10 \mathrm{~B})$ & 109.5 \\
\hline $\mathrm{H}(10 \mathrm{~A})-\mathrm{C}(10)-\mathrm{H}(10 \mathrm{~B})$ & 109.5 & $\mathrm{~N}(1)-\mathrm{C}(10)-\mathrm{H}(10 \mathrm{C})$ & 109.5 \\
\hline $\mathrm{H}(10 \mathrm{~A})-\mathrm{C}(10)-\mathrm{H}(10 \mathrm{C})$ & 109.5 & H(10B)-C(10)-H(10C) & 109.5 \\
\hline $\mathrm{N}(2)-\mathrm{C}(11)-\mathrm{O}(2)$ & $124.28(9)$ & $\mathrm{N}(2)-\mathrm{C}(11)-\mathrm{C}(12)$ & $126.62(9)$ \\
\hline $\mathrm{O}(2)-\mathrm{C}(11)-\mathrm{C}(12)$ & $109.10(8)$ & $\mathrm{C}(13)-\mathrm{C}(12)-\mathrm{C}(11)$ & $107.34(9)$ \\
\hline$C(13)-C(12)-C(15)$ & $130.90(9)$ & $C(11)-C(12)-C(15)$ & $121.75(9)$ \\
\hline$C(12)-C(13)-C(14)$ & $107.55(9)$ & $\mathrm{C}(12)-\mathrm{C}(13)-\mathrm{C}(19)$ & $131.01(10)$ \\
\hline$C(14)-C(13)-C(19)$ & $121.43(9)$ & $\mathrm{O}(3)-\mathrm{C}(14)-\mathrm{O}(2)$ & $120.68(10)$ \\
\hline $\mathrm{O}(3)-\mathrm{C}(14)-\mathrm{C}(13)$ & $130.40(10)$ & $\mathrm{O}(2)-\mathrm{C}(14)-\mathrm{C}(13)$ & $108.91(8)$ \\
\hline
\end{tabular}




$\begin{array}{ll}\mathrm{C}(12)-\mathrm{C}(15)-\mathrm{C}(16) & 112.43(8) \\ \mathrm{C}(16)-\mathrm{C}(15)-\mathrm{H}(15 \mathrm{~A}) & 109.1 \\ \mathrm{C}(16)-\mathrm{C}(15)-\mathrm{H}(15 \mathrm{~B}) & 109.1 \\ \mathrm{C}(17)-\mathrm{C}(16)-\mathrm{C}(18) & 111.03(11) \\ \mathrm{C}(18)-\mathrm{C}(16)-\mathrm{C}(15) & 111.05(9) \\ \mathrm{C}(18)-\mathrm{C}(16)-\mathrm{H}(16) & 108.5 \\ \mathrm{C}(16)-\mathrm{C}(17)-\mathrm{H}(17 \mathrm{~A}) & 109.5 \\ \mathrm{H}(17 \mathrm{~A})-\mathrm{C}(17)-\mathrm{H}(17 \mathrm{~B}) & 109.5 \\ \mathrm{H}(17 \mathrm{~A})-\mathrm{C}(17)-\mathrm{H}(17 \mathrm{C}) & 109.5 \\ \mathrm{C}(16)-\mathrm{C}(18)-\mathrm{H}(18 \mathrm{~A}) & 109.5 \\ \mathrm{H}(18 \mathrm{~A})-\mathrm{C}(18)-\mathrm{H}(18 \mathrm{~B}) & 109.5 \\ \mathrm{H}(18 \mathrm{~A})-\mathrm{C}(18)-\mathrm{H}(18 \mathrm{C}) & 109.5 \\ \mathrm{C}(13)-\mathrm{C}(19)-\mathrm{H}(19 \mathrm{~A}) & 109.5 \\ \mathrm{H}(19 \mathrm{~A})-\mathrm{C}(19)-\mathrm{H}(19 \mathrm{~B}) & 109.5 \\ \mathrm{H}(19 \mathrm{~A})-\mathrm{C}(19)-\mathrm{H}(19 \mathrm{C}) & 109.5\end{array}$

$\begin{array}{ll}\mathrm{C}(12)-\mathrm{C}(15)-\mathrm{H}(15 \mathrm{~A}) & 109.1 \\ \mathrm{C}(12)-\mathrm{C}(15)-\mathrm{H}(15 \mathrm{~B}) & 109.1 \\ \mathrm{H}(15 \mathrm{~A})-\mathrm{C}(15)-\mathrm{H}(15 \mathrm{~B}) & 107.8 \\ \mathrm{C}(17)-\mathrm{C}(16)-\mathrm{C}(15) & 109.21(10) \\ \mathrm{C}(17)-\mathrm{C}(16)-\mathrm{H}(16) & 108.5 \\ \mathrm{C}(15)-\mathrm{C}(16)-\mathrm{H}(16) & 108.5 \\ \mathrm{C}(16)-\mathrm{C}(17)-\mathrm{H}(17 \mathrm{~B}) & 109.5 \\ \mathrm{C}(16)-\mathrm{C}(17)-\mathrm{H}(17 \mathrm{C}) & 109.5 \\ \mathrm{H}(17 \mathrm{~B})-\mathrm{C}(17)-\mathrm{H}(17 \mathrm{C}) & 109.5 \\ \mathrm{C}(16)-\mathrm{C}(18)-\mathrm{H}(18 \mathrm{~B}) & 109.5 \\ \mathrm{C}(16)-\mathrm{C}(18)-\mathrm{H}(18 \mathrm{C}) & 109.5 \\ \mathrm{H}(18 \mathrm{~B})-\mathrm{C}(18)-\mathrm{H}(18 \mathrm{C}) & 109.5 \\ \mathrm{C}(13)-\mathrm{C}(19)-\mathrm{H}(19 \mathrm{~B}) & 109.5 \\ \mathrm{C}(13)-\mathrm{C}(19)-\mathrm{H}(19 \mathrm{C}) & 109.5 \\ \mathrm{H}(19 \mathrm{~B})-\mathrm{C}(19)-\mathrm{H}(19 \mathrm{C}) & 109.5 \\ & \end{array}$


Table 5. Anisotropic displacement parameters $\left(\AA^{2}\right)$ for 8 . The anisotropic displacement factor exponent takes the form: $-2 \pi^{2}\left[\mathrm{~h}^{2} \mathrm{a}^{* 2} \mathrm{U}_{11}+\ldots+2 \mathrm{~h} \mathrm{k} \mathrm{a} \mathrm{b}^{*} \mathrm{U}_{12}\right]$.

$\begin{array}{lllllll} & \mathrm{U}_{11} & \mathrm{U}_{22} & \mathrm{U}_{33} & \mathrm{U}_{23} & \mathrm{U}_{13} & \mathrm{U}_{12} \\ \mathrm{~N}(1) & 0.0184(3) & 0.0231(4) & 0.0157(3) & -0.0011(3) & 0.0023(3) & 0.0023(3) \\ \mathrm{N}(2) & 0.0181(3) & 0.0186(4) & 0.0146(3) & 0.0003(3) & 0.0023(2) & 0.0009(3) \\ \mathrm{O}(1) & 0.0297(4) & 0.0526(6) & 0.0149(3) & -0.0022(4) & 0.0009(3) & 0.0113(4) \\ \mathrm{O}(2) & 0.0165(3) & 0.0205(3) & 0.0167(3) & 0.0004(2) & 0.0025(2) & -0.0014(2) \\ \mathrm{O}(3) & 0.0193(3) & 0.0299(4) & 0.0278(4) & 0.0001(3) & 0.0055(3) & -0.0041(3) \\ \mathrm{C}(1) & 0.0174(4) & 0.0209(4) & 0.0140(3) & 0.0017(3) & 0.0023(3) & 0.0010(3) \\ \mathrm{C}(2) & 0.0182(4) & 0.0219(4) & 0.0227(4) & 0.0057(4) & 0.0044(3) & 0.0014(3) \\ \mathrm{C}(3) & 0.0175(4) & 0.0166(4) & 0.0263(4) & 0.0031(3) & 0.0030(3) & 0.0002(3) \\ \mathrm{C}(4) & 0.0227(5) & 0.0170(4) & 0.0290(5) & -0.0011(4) & 0.0034(4) & 0.0004(3) \\ \mathrm{C}(5) & 0.0280(5) & 0.0202(5) & 0.0368(6) & -0.0058(4) & 0.0092(4) & 0.0015(4) \\ \mathrm{C}(6) & 0.0214(5) & 0.0237(5) & 0.0492(7) & -0.0051(5) & 0.0091(5) & 0.0014(4) \\ \mathrm{C}(7) & 0.0188(5) & 0.0262(5) & 0.0450(7) & 0.0013(5) & 0.0008(4) & 0.0001(4) \\ \mathrm{C}(8) & 0.0197(4) & 0.0236(5) & 0.0298(5) & 0.0031(4) & 0.0002(4) & -0.0003(4) \\ \mathrm{C}(9) & 0.0192(4) & 0.0220(4) & 0.0156(4) & 0.0008(3) & 0.0030(3) & 0.0015(3) \\ \mathrm{C}(10) & 0.0225(5) & 0.0281(5) & 0.0243(4) & -0.0036(4) & 0.0052(3) & 0.0060(4) \\ \mathrm{C}(11) & 0.0171(4) & 0.0155(4) & 0.0161(3) & -0.0008(3) & 0.0019(3) & 0.0014(3) \\ \mathrm{C}(12) & 0.0188(4) & 0.0169(4) & 0.0152(3) & -0.0004(3) & 0.0028(3) & 0.0010(3) \\ \mathrm{C}(13) & 0.0205(4) & 0.0208(4) & 0.0170(4) & 0.0010(3) & 0.0039(3) & -0.0005(3) \\ \mathrm{C}(14) & 0.0195(4) & 0.0192(4) & 0.0196(4) & 0.0003(3) & 0.0053(3) & -0.0003(3) \\ \mathrm{C}(15) & 0.0186(4) & 0.0217(4) & 0.0162(4) & -0.0004(3) & 0.0010(3) & 0.0012(3) \\ \mathrm{C}(16) & 0.0242(4) & 0.0235(5) & 0.0184(4) & -0.0013(3) & 0.0019(3) & -0.0029(4) \\ \mathrm{C}(17) & 0.0337(6) & 0.0385(7) & 0.0389(7) & -0.0034(6) & -0.0105(5) & -0.0094(5) \\ \mathrm{C}(18) & 0.0426(7) & 0.0235(5) & 0.0279(5) & -0.0034(4) & 0.0130(4) & 0.0004(5) \\ \mathrm{C}(19) & 0.0291(5) & 0.0361(6) & 0.0198(4) & 0.0047(4) & 0.0076(4) & -0.0042(4)\end{array}$


Table 6. Hydrogen coordinates and isotropic displacement parameters $\left(\AA^{2}\right)$ for 8.

$\begin{array}{lllll} & \mathrm{x} & \mathrm{y} & \mathrm{z} & \mathrm{U}(\mathrm{eq}) \\ \mathrm{H}(1 \mathrm{~N}) & 1.011(3) & 0.1138(18) & 0.4365(18) & 0.034(4) \\ \mathrm{H}(1) & 0.5756 & 0.1442 & 0.3371 & 0.021 \\ \mathrm{H}(2 \mathrm{~A}) & 0.7629 & 0.3397 & 0.3702 & 0.025 \\ \mathrm{H}(2 \mathrm{~B}) & 0.6538 & 0.3078 & 0.2259 & 0.025 \\ \mathrm{H}(4) & 0.5862 & 0.3714 & 0.5704 & 0.027 \\ \mathrm{H}(5) & 0.3087 & 0.4220 & 0.6441 & 0.033 \\ \mathrm{H}(6) & 0.0405 & 0.4223 & 0.4908 & 0.037 \\ \mathrm{H}(7) & 0.0497 & 0.3712 & 0.2634 & 0.036 \\ \mathrm{H}(8) & 0.3254 & 0.3170 & 0.1905 & 0.030 \\ \mathrm{H}(10 \mathrm{~A}) & 1.2148 & 0.1233 & 0.2456 & 0.037 \\ \mathrm{H}(10 \mathrm{~B}) & 1.0880 & 0.0259 & 0.2019 & 0.037 \\ \mathrm{H}(10 \mathrm{C}) & 1.2201 & 0.0244 & 0.3432 & 0.037 \\ \mathrm{H}(15 \mathrm{~A}) & 0.9406 & 0.1190 & 0.7694 & 0.023 \\ \mathrm{H}(15 \mathrm{~B}) & 0.8446 & 0.1209 & 0.9073 & 0.023 \\ \mathrm{H}(16) & 0.8746 & 0.2987 & 0.7346 & 0.026 \\ \mathrm{H}(17 \mathrm{~A}) & 1.0742 & 0.2563 & 0.9967 & 0.057 \\ \mathrm{H}(17 \mathrm{~B}) & 1.0926 & 0.3605 & 0.9121 & 0.057 \\ \mathrm{H}(17 \mathrm{C}) & 1.1599 & 0.2534 & 0.8545 & 0.057 \\ \mathrm{H}(18 \mathrm{~A}) & 0.7613 & 0.3949 & 0.9144 & 0.046 \\ \mathrm{H}(18 \mathrm{~B}) & 0.7263 & 0.2893 & 0.9915 & 0.046 \\ \mathrm{H}(18 \mathrm{C}) & 0.6121 & 0.3147 & 0.8467 & 0.046 \\ \mathrm{H}(19 \mathrm{~A}) & 0.5566 & 0.0804 & 0.9823 & 0.042 \\ \mathrm{H}(19 \mathrm{~B}) & 0.4471 & -0.0165 & 0.9136 & 0.042 \\ \mathrm{H}(19 \mathrm{C}) & 0.3435 & 0.0905 & 0.9296 & 0.042\end{array}$


Fig 1. 1H NMR of 3

DHB-12

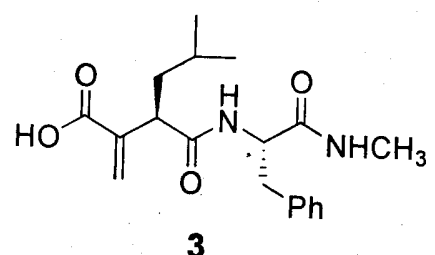

$\exp 1$ s2pul

\begin{tabular}{lll} 
SAMPLE & \multicolumn{1}{c}{ DEC. \& VT } \\
date Jan 292005 & dfrq & $\mathbf{4 9 9 . 8 6 4}$ \\
solvent & $\operatorname{CDC} 13$ & dn
\end{tabular}

file $\mathrm{CDCl}$ di dn

\begin{tabular}{rrlr}
\multicolumn{1}{c}{ ACQUISITION } & exp & dpwr & dof \\
sfrq & 499.864 & dm & 0 \\
tn & $H 1$ & dmm & nnn \\
at & 4.989 & dmf & 200
\end{tabular}

$\begin{array}{lrlr}\mathrm{np} & 65536 & \mathrm{dseq} & \\ \mathrm{sw} & 6568.1 & \text { dres } & 1.0 \\ \mathrm{fb} & 4000 & \text { homo } & \mathrm{n}\end{array}$

$\begin{array}{lrlll}\text { bs } & 8 & & \text { DEC2 } & \\ \text { tpwr } & 59 & \text { dfrq2 } & & 0\end{array}$

$\begin{array}{lrll}\text { pw } & 10.6 & \text { dn2 } & \\ \text { d1 } & 0.100 & \text { dpwr } 2 & 1 \\ \text { tof } & 300.3 & \text { dof } 2 & 0 \\ \text { nt } & 16 & \text { dm2 } & n\end{array}$

$\begin{array}{llll}\mathrm{nt} & 16 & \mathrm{dm} 2 & \mathrm{n} \\ \mathrm{ct} & 16 & \mathrm{dmm} 2 & \mathrm{c}\end{array}$

gain not used dseq2

$\begin{array}{llll}i 1 & n & 1.0 \\ \text { in } & n \text { homo2 } & & \\ & & \text { DEC3 } & n\end{array}$

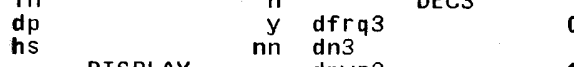

$\begin{array}{rrll}\mathrm{sp} & \text { DISPLAY } & \text { dpwr3 } & 1 \\ \mathrm{wp} & 217.2 & \text { dof } 3 & 0 \\ \mathrm{~s} & 5851.6 & \mathrm{dm} 3 & n \\ \mathrm{sc} & 109 & \mathrm{dmm} 3 & \mathrm{c}\end{array}$

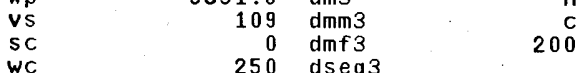

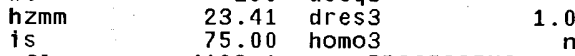

$\begin{array}{lll}\text { is } & 75.00 & \text { hOmO3 } \\ \text { if } 1 & 4128.1 & \text { PROCESSING }\end{array}$

$\begin{array}{lrlr}\text { rfp } & 3629.0 & \text { wtfile } & \\ \text { th } & & \text { proc } & \mathrm{ft} \\ \text { ins } & 5.000 & \text { fn } & 65536\end{array}$

werr
wexp process plH
wbs
wht

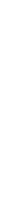

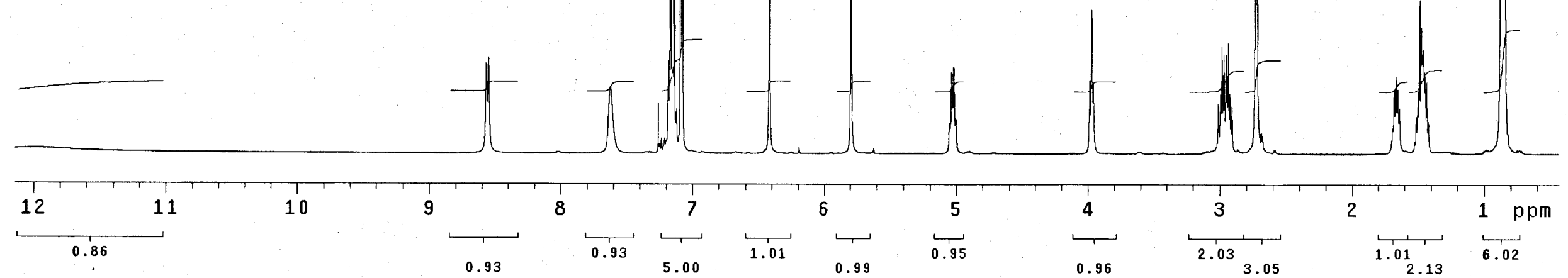


DHB-12

Pulse Sequence: relayh Solvent: $\operatorname{CDC} 13$ Ambient temperature
INOVA-500 "inova5"

Re lax. de lay $1.300 \mathrm{sec}$ Cosy $90-90$
Acq. time $0.247 \mathrm{sec}$
Width $4152.2 \mathrm{~Hz}$ $\begin{array}{ll}\text { Width } & 4152.2 \mathrm{~Hz} \\ 2 \mathrm{D} \text { width } & 4152.2 \mathrm{~Hz}\end{array}$ 4 repetitions

OBSERVE H1, 499.8611767 MHz DATA PROCESSING

Sine be $110.123 \mathrm{sec}$

Sine be 110 OCSING

FT size $2048 \times 2048$

Total time $55 \mathrm{~min}, 27 \mathrm{sec}$

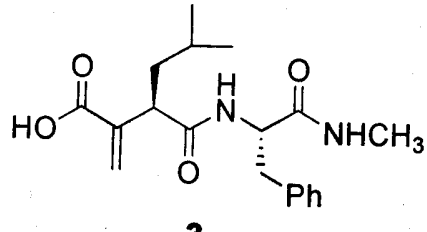

3
Fig 2. $1 \mathrm{H}-1 \mathrm{H}$ NHM of 3

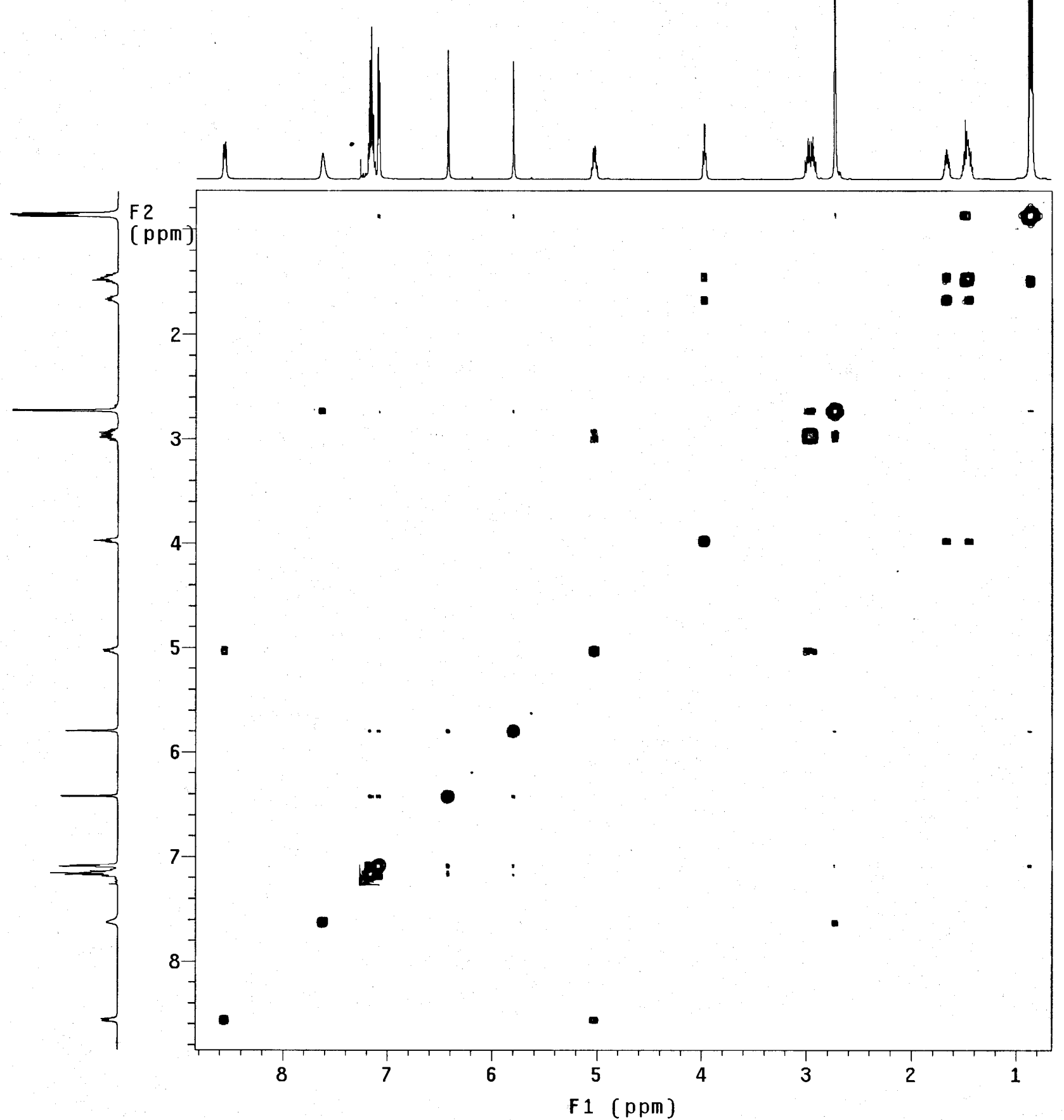


Fig 3. $13 \mathrm{C}$ NMR of 3

DHB-12

exp3 s2pul

SAMPLE
Jan 292005 dfrq

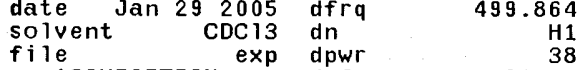

$\begin{array}{rrrr}\text { fi le } & \text { exp } & \text { dpwr } & 38 \\ \text { ACQUISITION } & \text { dof } & 300.3 \\ \text { sfrq } & 125.704 & \text { dm } & \text { YYy }\end{array}$

$\begin{array}{lrlr}\mathrm{sfrq} & 125.704 & \mathrm{dm} & \mathrm{YHY} \\ \mathrm{tn} & \mathrm{C13} & \mathrm{dmm} & \mathrm{W} \\ \text { at } & 1.153 & \mathrm{dmf} & 9901\end{array}$

$\begin{array}{rrrr}\text { at } & 11.153 & \text { dmf } & 9901 \\ \text { np } & 65536 & \text { dseq } & 1.0 \\ \text { fw } & 28429.3 & \text { dres } & 1.0 \\ \text { fb } & 16000 & \text { homo } & \end{array}$

$\begin{array}{lrlll}\mathrm{fb} & 16000 & \text { homo } & & \mathrm{N} \\ \mathrm{bs} & 4 & & \text { DEC2 } & \\ \mathrm{tpwr} & 52 & \mathrm{dfrq2} & & 0\end{array}$

$\begin{array}{lrl}\text { pw } & 11.0 & \text { dn2 } \\ \text { d1 } & 2.000 & \text { dpwr2 }\end{array}$

$\begin{array}{lrlr}\text { d1 } & 2.000 & \text { dpwr } 2 & 1 \\ \text { tof } & 1670.1 & \text { dof2 } & 0 \\ \text { nt } & 640 & \text { dm2 } & n \\ \text { ct } & 108 & \text { dmm2 } & c \\ \text { a lock } & n & \text { dmf2 } & 10000\end{array}$

gain not used dseq2

$\begin{array}{lllll}\text { i1 } & \text { FLAGS } & \text { n dres2 } & & 1.0 \\ \text { in } & & n & & \\ & & \text { DEmo2 } & \text { DEC3 } & \end{array}$

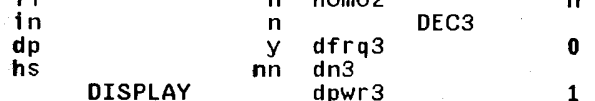

$\begin{array}{lrll}\text { sp } & \text { DISPLAY } & \text { dpwr3 } & 1 \\ \text { wp } & 2461.0 & \text { dof } 3 & 0 \\ \text { vs } & 20172.4 & \text { dm3 } & n \\ \text { s } & 153 & \text { dmm3 } & c\end{array}$

$\begin{array}{rrrr}\text { vs } & 201753 & \text { dm3 } & n \\ s c & 153 & \text { dmm3 } & c \\ \text { dmf } & 0 & \text { dmf } 3 & 10000\end{array}$

$\begin{array}{rrrrr}\text { SC } & 0 & \text { dmf } 3 & 10000 & \text { dseq3 }\end{array}$

$\begin{array}{lrrr}\text { hzmm } & 80.69 & \text { dres3 } & 1.0 \\ \text { is } & 500.00 & \text { homo3 } & 1.0\end{array}$

rfl 102.5 PROCESSING

$\begin{array}{lll}\mathrm{rfp} & 0 & \mathrm{lb} \\ \mathrm{th} & 5 & \text { wtfile }\end{array}$

$\begin{array}{lllr}\text { th } & & 100.000 & \begin{array}{l}\text { wtfile } \\ \text { proc }\end{array} \\ \text { ai cdc ph } & \text { fn } & \text { math } & 65536 \\ & & & \text { math }\end{array}$

$\mathrm{ft}$
536
$\mathrm{f}$

werr
wexp process pic

Wht $\quad$ Whs
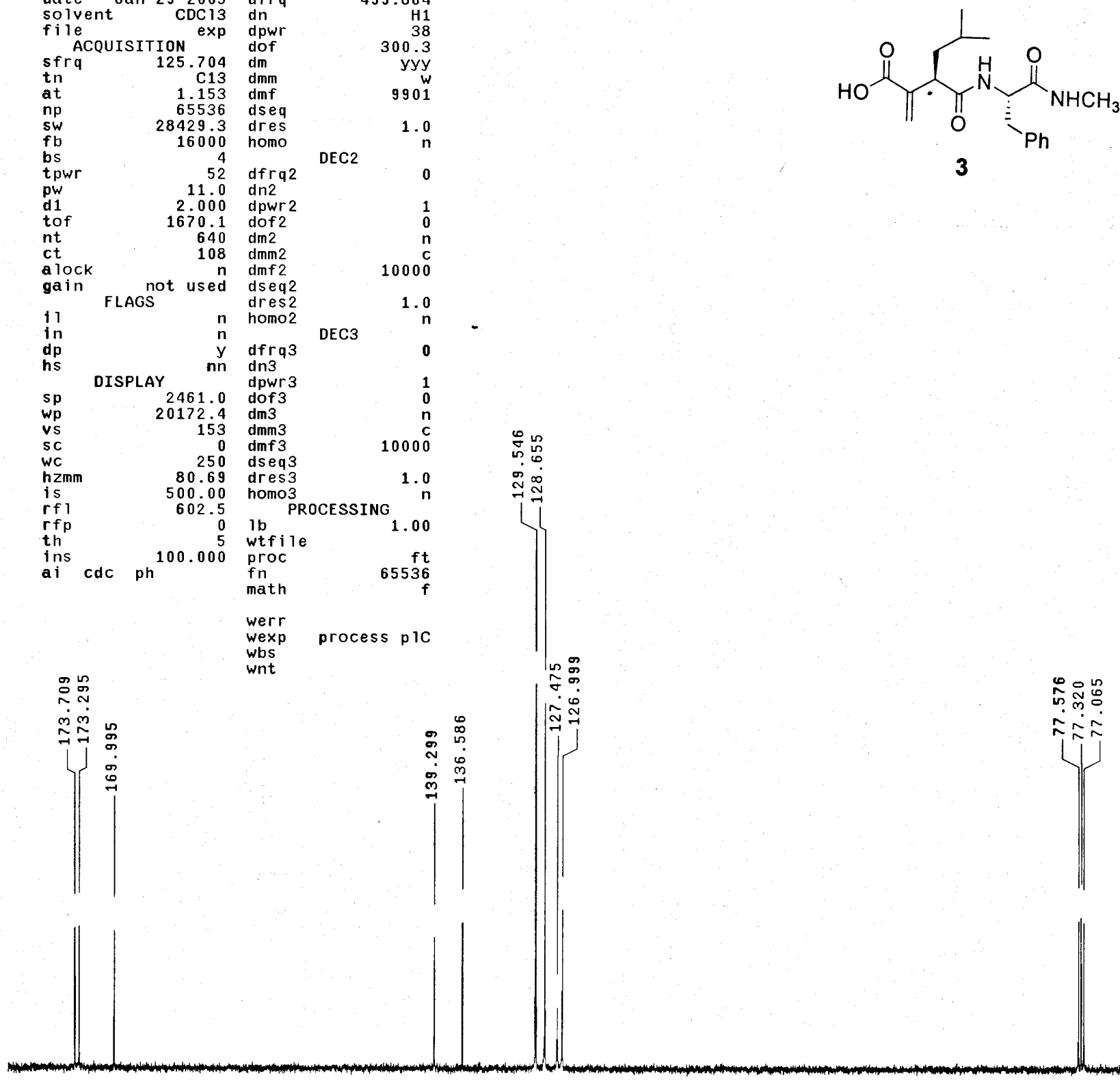


\section{ATLANTIC MICROLAB, INC.}

Sample No. DHB-29

\section{P.O. Box 2288}

Norcross, Georgia 30091

(770) 242-0082

\section{rationtionicosob.com}

PROFESSOR/SUPERVISOR:

P.O. \#:

\begin{tabular}{c|c|c|c}
\hline Element & Theory & \multicolumn{2}{|c}{ Found } \\
\hline $\mathrm{C}$ & 69.49 & 69.74 & \\
\hline $\mathrm{H}$ & 7.37 & 7.45 & \\
\hline $\mathrm{N}$ & 8.53 & 8.50 & \\
\hline & & & \\
\hline & & & \\
\hline
\end{tabular}

Date Received APR 152005 Date Completed
Company/School Notre Dame University

Address Department of Chemistry and Biochemistry 419b Nieuwland Science Center, Notre Dame, IN 46556

NAME Dr. Dusan Hesek DATE

\begin{tabular}{|c|c|}
\hline Single 龱 & Duplicate $\square$ \\
\hline $\begin{array}{l}\text { Elements } \\
\text { Present: }\end{array}$ & $\mathrm{C}, \mathrm{H}, \mathrm{N}, \mathrm{O}$ \\
\hline $\begin{array}{l}\text { Analyze } \\
\text { for: }\end{array}$ & $\mathrm{C}, \mathrm{H}, \mathrm{N}$ \\
\hline $\begin{array}{l}\text { Hygroscopic }[ \\
\text { M.P. }\end{array}$ & $\begin{array}{r}\text { Explosive } \square \\
\text { B.P. } \\
\end{array}$ \\
\hline $\begin{array}{l}\text { To be dried: Ye } \\
\text { Temp. } \\
\text { FAX Service [ } \\
\text { FAX Phone \# }\end{array}$ & 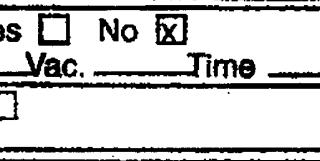 \\
\hline $\begin{array}{l}\text { Rush Service } \\
\text { Phone Service } \\
\text { Phone No. }\end{array}$ & $\begin{array}{ll} & \text { (SEE CURRENT } \\
\square & \text { PRICE LIST) } \\
\end{array}$ \\
\hline
\end{tabular}

Phone No.

Remarks:

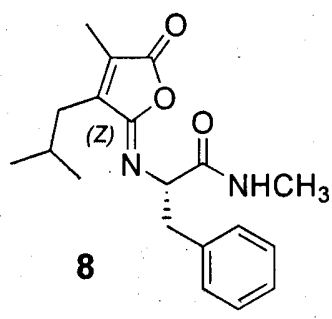


Fig 4. $1 \mathrm{H}$ NMR of 8

exp1 s2pur

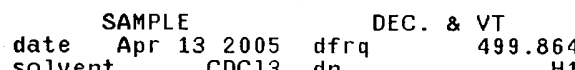

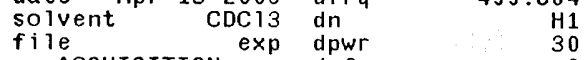

ACQUISITION $\exp$ dof

$\begin{array}{lrlr}\mathrm{tn} & \mathrm{H} 1 & \mathrm{dmm} & \mathrm{c} \\ \mathrm{ta} & 2.902 & \mathrm{dmf} & 200\end{array}$

$\begin{array}{rrrr}\text { at } & 2.902 & \text { dmf } & \\ \text { np } & 32768 & \text { dseq } & \\ \text { sw } & 5645.3 & \text { dres } & 1.0 \\ \text { fb } & 3000 & \text { homo } & \end{array}$

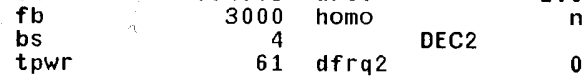

pw 13.5 dn2

$\begin{array}{lrll}\text { d1 } & 1.000 & \text { dpwr } & 1 \\ \text { tof } & -210.2 & \text { dof 2 } & 0 \\ \text { nt } & 16 & \text { dm2 } & n \\ \text { ct } & 16 & \text { dmm } & \end{array}$

$\begin{array}{lrr}\mathrm{ct} & 16 & \operatorname{dmm} 2 \\ \text { a lock } & \mathrm{n} & \text { dmf2 }\end{array}$

gain not used dseq2 200

i1 FLAGS $n$ hres2 1.0

$\begin{array}{lrlll}\text { in } & n & \text { homo2 } & \text { DEC3 } & n \\ d p & y & d f r q 3 & & 0 \\ \text { hs } & y n & d n 3 & & \end{array}$

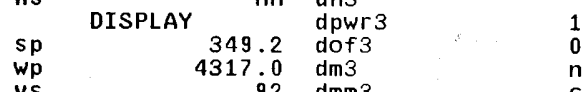

$\begin{array}{lrll}\text { VS } & 92 & d m m 3 \\ \text { SC } & 0 & d m f 3\end{array}$

$\begin{array}{lrrr}\text { WC } & 250 & \text { dseq3 } & \\ \text { hzmm } & 17.27 & \text { dres3 } & 1.0\end{array}$

is $\quad 111.41$ homo3

$\begin{array}{rrr}r f 1 & 533.6 & \text { PROCESSING } \\ r f p & 0 & \text { wtfile }\end{array}$

th 54 wtfi

ins ph 4.000 fn

$\begin{array}{lr}f n & f t \\ \text { math } & 32768\end{array}$

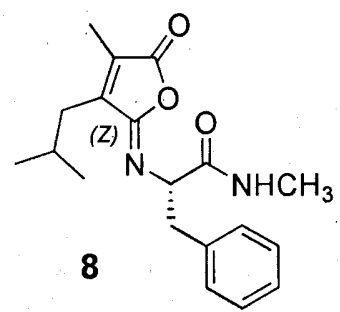

werr
wexp process $p 1 H$
wbs

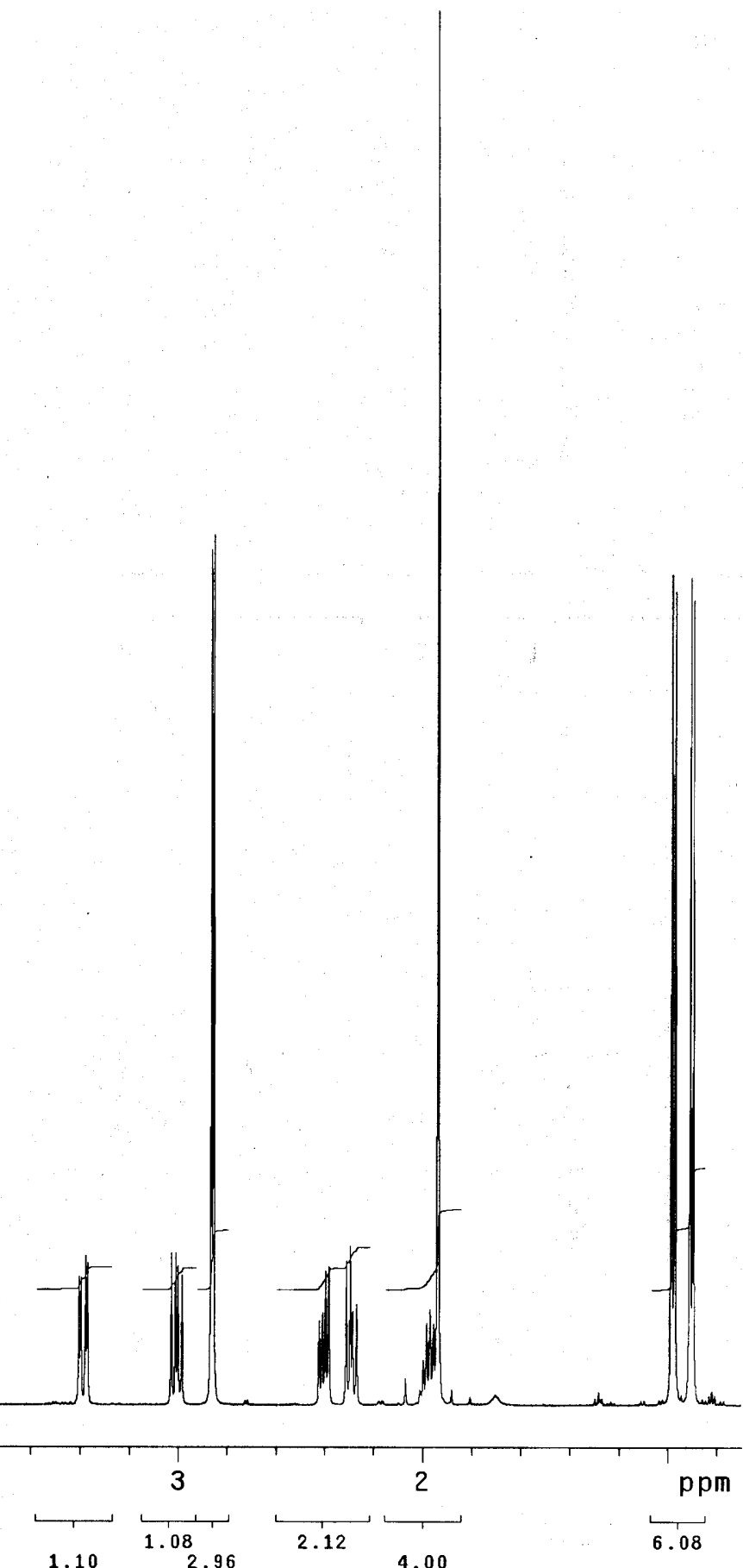

$\mathrm{S} 13$ 
DHB-29

Pulse Sequence: relayh

Solvent: $\operatorname{CDC} 13$

Ambient temperature
INOVA-500 "inova5"

Re lax. de lay $1.300 \mathrm{sec}$

COSY $90-900.149 \mathrm{sec}$

Acq. time $0.149 \mathrm{sec}$
Width $3442.5 \mathrm{~Hz}$

2D Width $3442.5 \mathrm{~Hz}$

16 repetitions

OBSERVE increments

DATA PROCESSING

Sine be $110.074 \mathrm{se}$

Sine bell 0.037 sec

Total time $1 \mathrm{hr}, 42 \mathrm{~min}, 19 \mathrm{sec}$

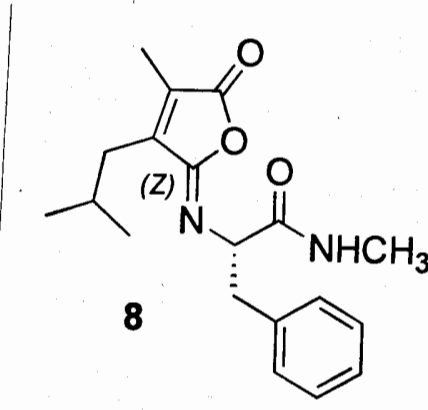

Fig 5. $1 \mathrm{H}-1 \mathrm{H} \mathrm{NMR}$ of 8

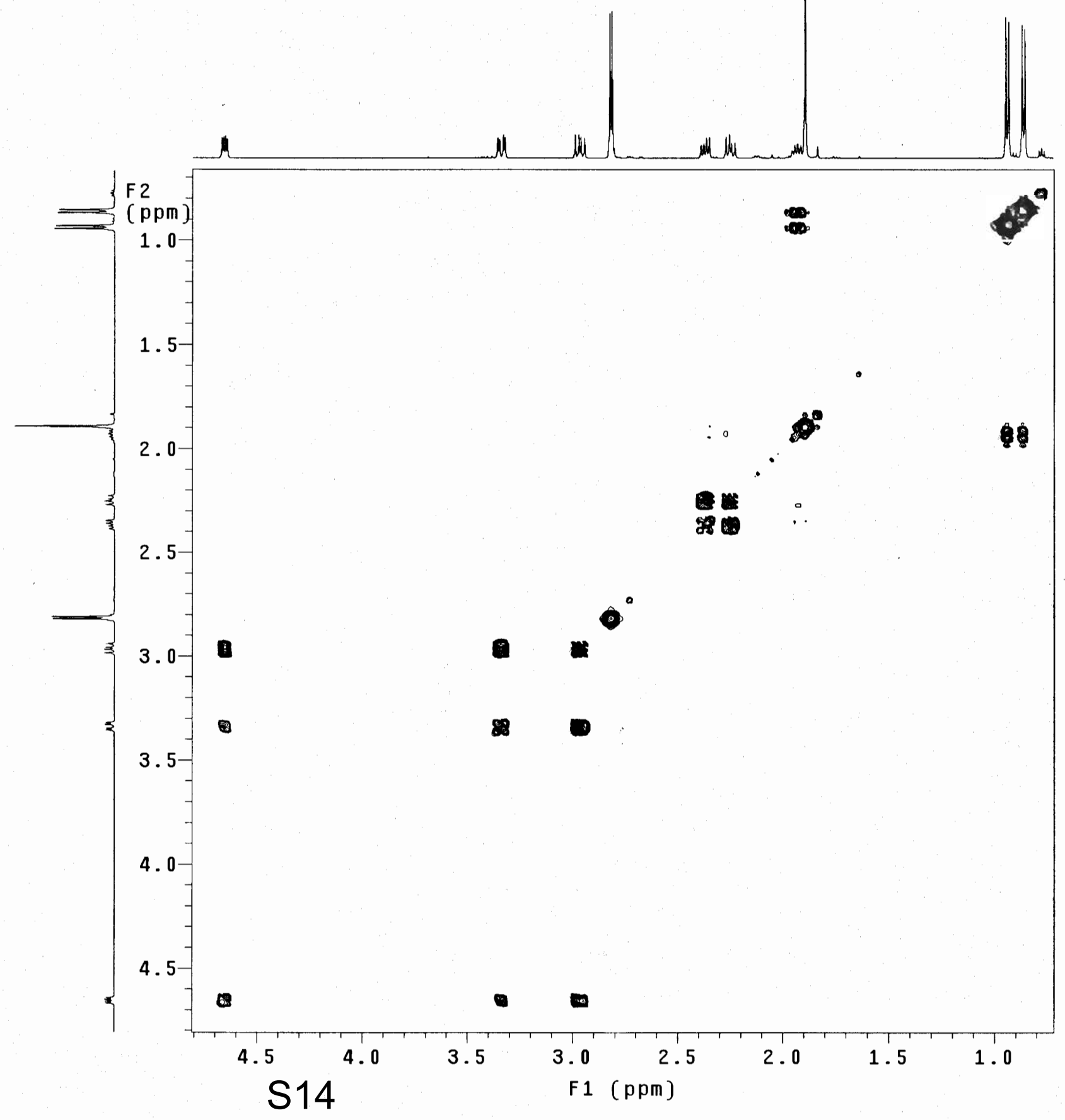


DHB-29

exp4 s2pur

date SAMPLE $\begin{gathered}\text { Dan } 282006 \text { dfrq } \\ \text { DEC. \& VT } \\ 499.864\end{gathered}$

$\begin{array}{llll}\text { date Jan } 282006 & \text { dfrq } & 499.864 \\ \text { solvent } \operatorname{CDC} 13 & \text { dn } & H 1\end{array}$

file /afs/nd.edu/ur dpwr

ser26/dhesek/Privar dof

te/DHB/DHB-29C.fid dm

$\begin{array}{lll}\text { sfrq } & 125.702 & \mathrm{dm} \\ \mathrm{tm} & & \mathrm{dm}\end{array}$

$\begin{array}{ll}\text { dmf } & 8787.35\end{array}$

$\begin{array}{lll}\text { at } & 1.215 & \begin{array}{l}\text { dseq } \\ \text { dres }\end{array} \\ \text { ap } & 1.0\end{array}$

$\begin{array}{lrlll}n p & 65536 & \text { homo } & & n \\ \mathrm{sw} & 26963.3 & & \mathrm{DEC} 2 & \end{array}$

$\begin{array}{rrrr}\text { fb } & 15000 & \text { dfrq2 } & 0 \\ \text { bs } & 64 & \text { dn2 } & \end{array}$

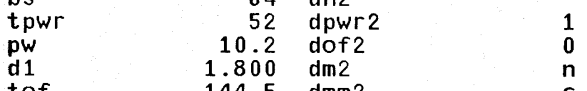

$\begin{array}{lrlr}\text { tof } & 144.5 & \mathrm{dmm} 2 & c \\ \mathrm{nt} & 1200 & \mathrm{dmf} 2 & 10000\end{array}$

$\begin{array}{lrlr}\text { ct } & 1200 & \text { dmeq2 } & \\ \text { a lock } & 256 & \text { dses2 } & 1.0\end{array}$

gain not used homo2 0 n

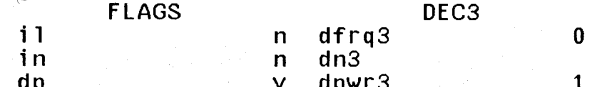

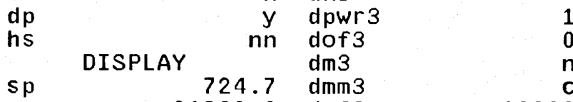

$\begin{array}{lrlr}\text { sp } & 724.7 & \text { dmm3 } & \\ \text { wp } & 21892.0 & \text { dmf } 3 & 10000 \\ \text { vs } & 243 & \text { dseq3 } & \\ \text { sc } & 0 & \text { dres3 } & 1.0 \\ \text { wC } & 250 & \text { homo3 } & \end{array}$

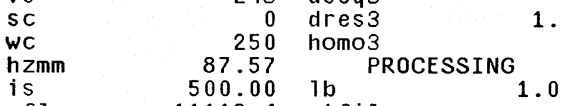

$\begin{array}{rrrr}r f l & 11118.4 & \text { wtfile } & f t \\ r f p & 9678.1 & \text { proc } & 131072\end{array}$

th 5 fn

ai cdc ph 100.000 math

math 131072

werr

wexp
whs
wnt

菅商
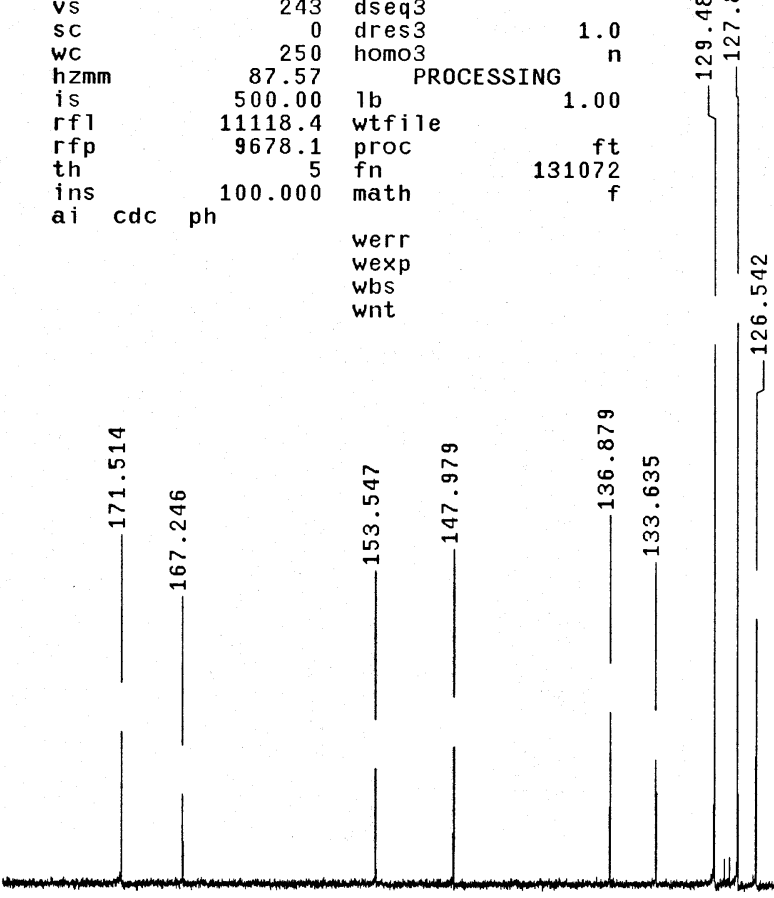

140

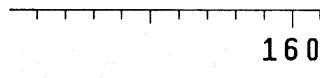

Fig 6. 13C NMR of 8

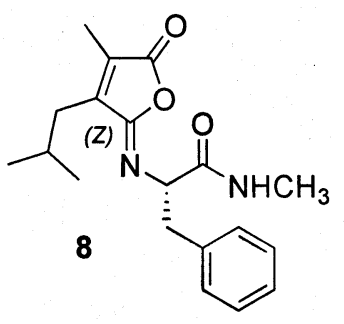

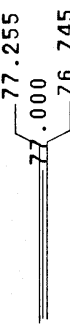
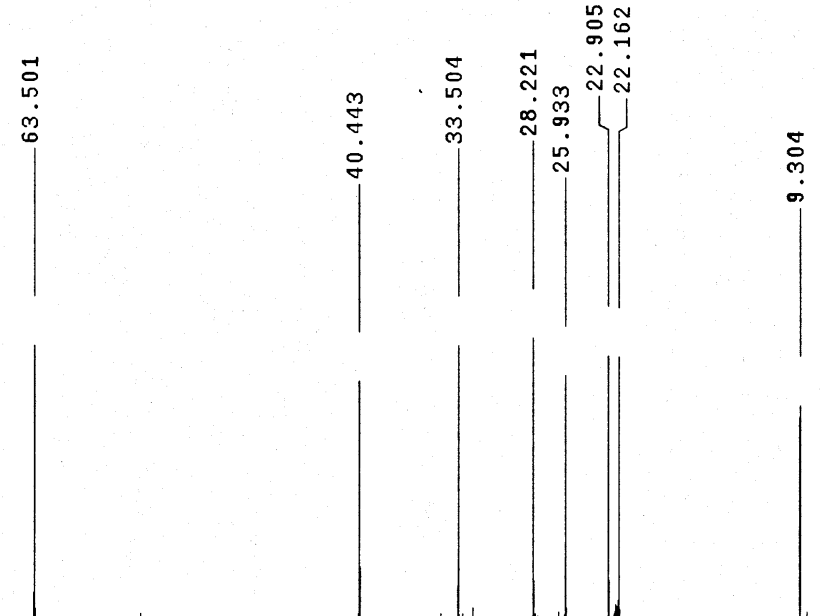


\section{DEPT NMR of 8}

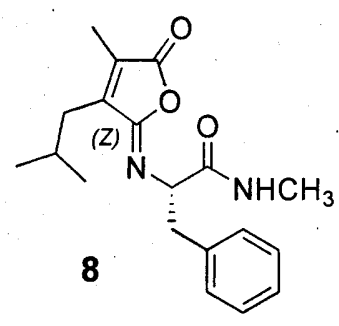

CH3 carbons
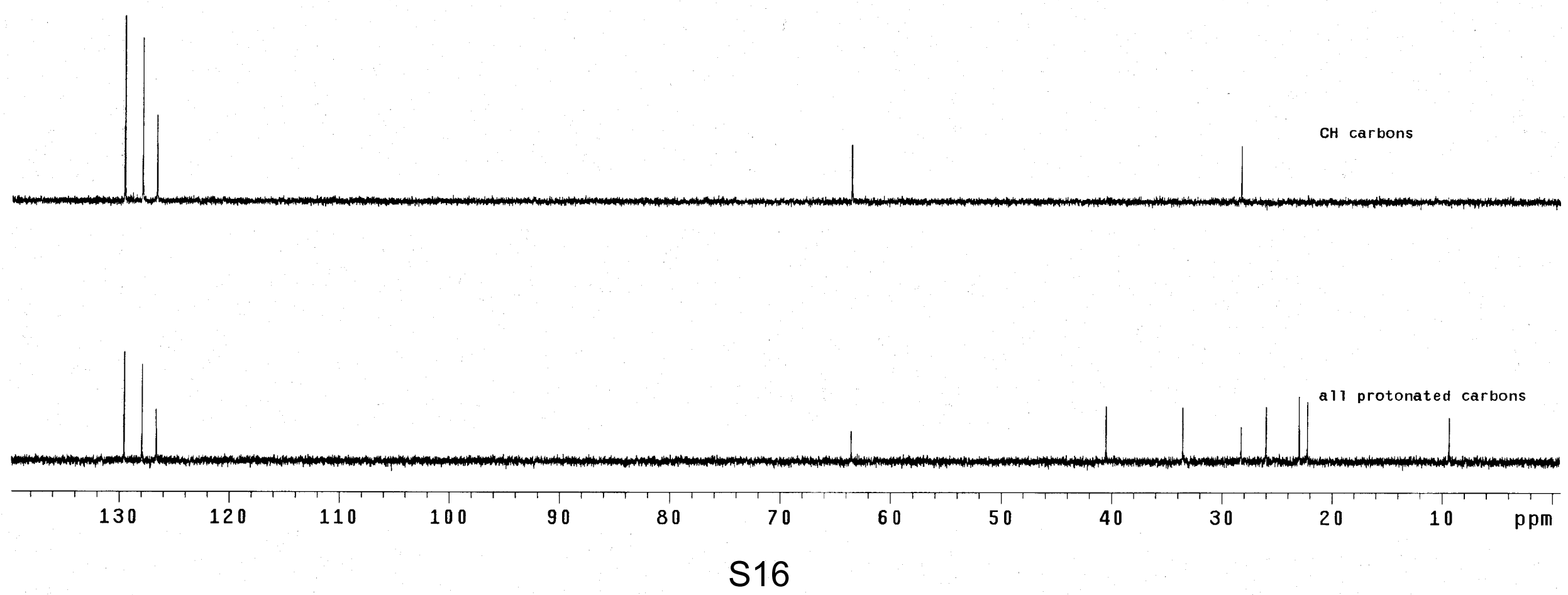


\section{General Experimental Methods}

Compounds 2-5 were prepared by published literature methods. ${ }^{21,22}$ MS electrospray of the purified derivatives were carried out using a Pro C18 YMC reverse phase column (3 x $50 \mathrm{~mm}$ ). The mobile phase was $10 \mathrm{mM}$ ammonium acetate in HPLC grade water (A), and HPLC grade acetonitrile (B). A gradient was formed from $5 \%$ to $80 \%$ of B in 10 minutes, and the flow rate of the LC was $0.7 \mathrm{~mL} / \mathrm{min}$. The MS electrospray source operated at capillary voltage $3.5 \mathrm{kV}$ and a desolvation temperature $300{ }^{\circ} \mathrm{C}$. The optical purity of synthesized derivatives was determined by a chiral reversed phase high performance liquid chromatography (RP-HPLC) analysis. The HPLC was performed using $(S, S)$ Whelk-O 1, Regis Technology column (250 x 4.6 mm, L x I.D.). An isocratic condition of mixed solvents system (hexane/ $\mathrm{CH}_{2} \mathrm{Cl}_{2} / \mathrm{EtOH} / \mathrm{AcOH}=70: 20: 10: 0.1$, v/v) was used for $15 \mathrm{~min}$, and the mobile-phase flow rate of the HPLC pump was $0.8 \mathrm{~mL} / \mathrm{min}$. A $6-\mu \mathrm{L}$ of sample solution was subject to HPLC for analysis and then taken the retention data at room temperature.

(21) Campion, C.; Davidson, A. H.; Dickens, J. P.; Crimmin, M. J. In PCT Int. Appl.; (1990) British Bio-Technology Ltd., UK, WO9005719.

(22) Sakamoto, M.; Imaoka, T.; Motoyama, M.; Yamamoto, Y.; Takasu, H. In PCT Int. Appl.; (1994) Otsuka Pharmaceutical Co., Ltd., Japan, WO 9421612. 